\title{
Acute Clinical Presentation of Metastatic Testicular Choriocarcinoma: A Case Report and Brief Review of Literature
}

\author{
Lakshmi Kant Pathak $^{\mathrm{a}, \mathrm{f}}$, Vimala Vijayaraghavan ${ }^{\mathrm{b}}$, Timothy Ponder ${ }^{\mathrm{c}}$, Niels-Jorgen Dyrved $^{\mathrm{d}}$, Darshan Gandhi ${ }^{\mathrm{e}}$
}

\begin{abstract}
We present an unusual case of a 23-year-old Hispanic male with no past medical history, who presented with 2 days history of diffuse abdominal pain. CT scan showed widely bleeding metastatic lesions in the retroperitoneum, lungs and liver causing large hemothorax and hemoperitoneum. He developed acute symptomatic anemia and needed interventional radiology-guided embolization of hepatic artery in addition with an urgent chest tube to relieve the symptoms along with transfusion. Subsequent exam found a true choriocarcinoma of the right testis. Following chemotherapy, the patient's lesions shrunk and his anemia improved. A review of literature shows that testicular choriocarcinoma is a rare aggressive malignancy which metastasizes hematogenously and bleeds extensively due to their high vascularity. They can present with acute symptoms and signs that often lead patients to seek emergency care. Acute bleeding manifestation could be also the first presentation often leading to the final diagnosis of choriocarcinoma like in this case.
\end{abstract}

Keywords: Choriocarcinoma; Hemothorax; Hemoperitoneum; Acute anemia

\section{Introduction}

Testicular germ cell tumors (GCTs) are rare representing 1-2\% of all malignancies in males. However, these tumors represent the most common malignancy in men aged $15-35$ years. GCT is classified into several subtypes, including seminoma (40\%),

Manuscript accepted for publication September 12, 2014

aDepartment of Hospital Medicine, Methodist Charlton Hospital, Dallas, TX, USA

${ }^{\mathrm{b}}$ Caribbean Medical University, USA

${ }^{\mathrm{c}}$ Radiology, Methodist Charlton Hospital, Dallas, TX, USA

dPathology, Methodist Charlton Medical Center, Dallas, TX, USA

eHematology-Oncology, Methodist Charlton Medical Center, Dallas, TX, USA fCorresponding Author: Lakshmi Kant Pathak, Department of Hospital Medicine, Methodist Charlton Hospital, 4613 Copper Mountain Lane, Richardson, Dallas, TX 75082, USA. Email: drpathaklk@gmail.com.

doi: http://dx.doi.org/10.14740/jmc1943w embroynal carcinoma $(25 \%)$, teratocarcinoma $(25 \%)$, teratoma $(5 \%)$ and pure choriocarcinoma $(1 \%)$.

Although the malignancy is more common in whites than nonwhites, there is no racial difference seen in choriocarcinoma. The tumor occurs most commonly in men aged $20-30$ years. It is an extremely rare type of testicular tumor.

Unlike other cancers, choriocarcinoma metastasizes hematogenously, with the testicular primary tumor often small or even "burned-out". Pure choriocarcinoma is more likely to manifest as symptoms of metastatic disease and is the most common element observed in brain metastases.

The local tumor in choriocarcinoma may be small or nonpalpable, whereas most testicular GCTs cause scrotal swelling and a palpable mass. Physical examination findings from lung, liver, and/or brain metastases may be more pronounced than an abnormal finding on testicular examination.

Beta HCG secreted by the synctiotrophoblastic cells is used as a tumor marker for diagnosis, grading and treatment response.

Patient can present with life threatening bleeding from highly vascular metastatic lesions as initial presentation and a successful management depends on treatment of underlying malignancy in addition to resuscitative measures.

Physicians should have a high degree of suspicion and be aware of specific imaging modalities and lab tests such as LDH, AFP and beta HCG to identify the diagnosis and expedite the treatment.

\section{Case Report}

A 23-year-old Hispanic male with no significant medical history presented to the Emergency Department with diffuse abdominal pain associated with nausea and vomiting. He also noticed a swelling in his right testicle, but did not seek medical advice as it was asymptomatic. The abdominal pain started a week ago especially worsened on physical activity. Patient is an athlete and plays soccer and it was particularly painful during play. Physical exam was normal, besides a small nontender right testicular mass. Retrospectively, the patient also reported that he noticed some gynecomastia a year ago but ignored it.

Initial evaluation including a CT of abdomen (non-contrast) showed multiple hepatic lesions with hemorrhage, larg- 


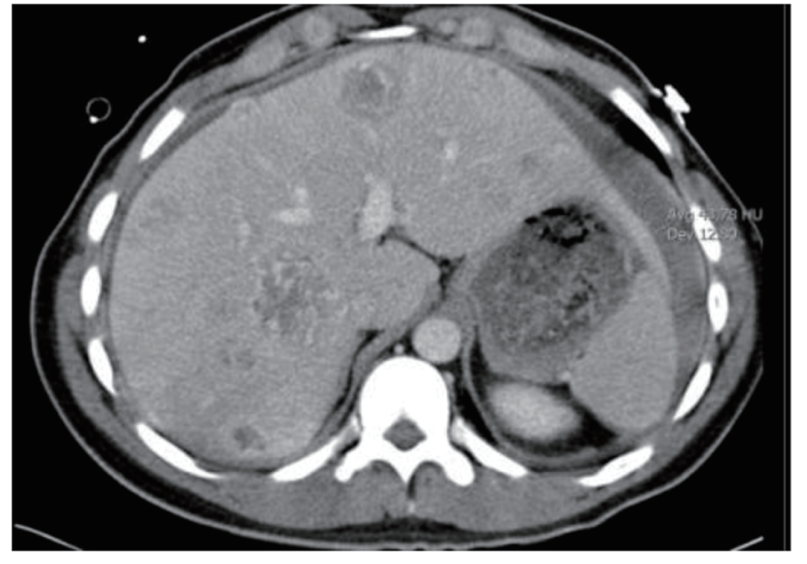

Figure 1. Liver Mets: choriocarcinoma metastatic to liver.

est $4 \mathrm{~cm}$ in dimension along with hemoperitoneum and bulky retroperitoneal and common iliac lymphadenopathy causing mild right hydronephrosis (Fig. 1,2). There were also multiple pulmonary nodules. Initial labs including $\mathrm{CBC}$ and biochemistry at the time of admission were mostly unremarkable (Table 1). Follow-up investigations included tumor markers which were grossly elevated and highly suggestive of a choriocarcinoma of testes (Table 2).

These finding were highly suspicious for testicular cancer in a young male (Table 2, Figs. 1-3).

The following day the patient showed signs of decompensation with hypotension and tachycardia. Repeat CT of chest showed a large pleural effusion suggestive of hemothorax (Fig. 3). The hemoglobin dropped to $6 \mathrm{~g}$ in $24 \mathrm{~h}$ and then he was symptomatic with dyspnea. A chest tube was inserted to relieve his dyspnea and $750 \mathrm{~mL}$ blood was immediately drained. Interventional radiology (IR) was consulted to stop

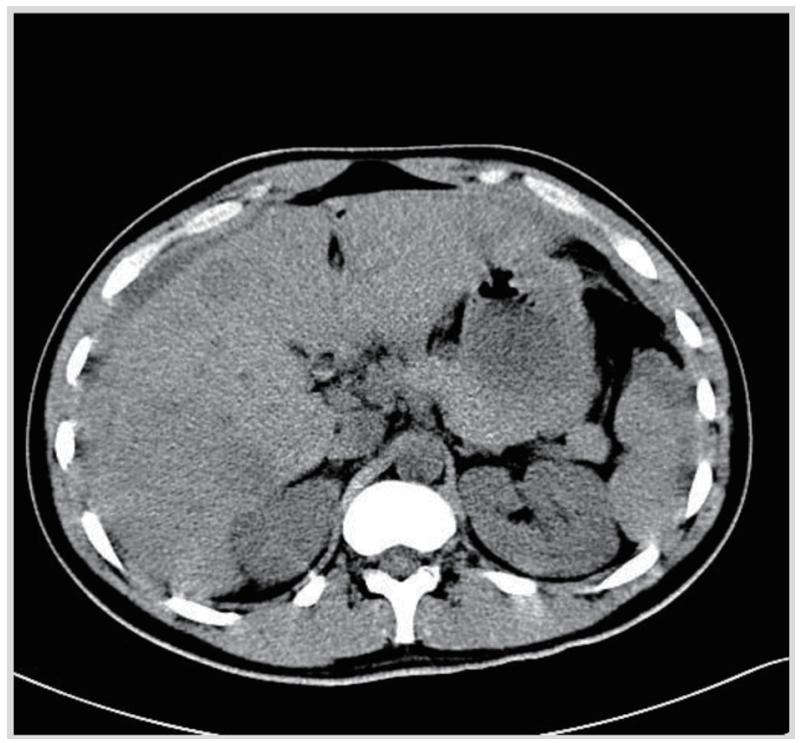

Figure 2. CT scan of abdomen and liver showing hemorrhagic metastasis.
Table 1. Initial Labs of the Patient on Admission

\begin{tabular}{ll}
\hline CBC & WBC 13, Hb 14, hematocrit 39 and platelets 192,000 \\
Biochemistry & Normal \\
\hline
\end{tabular}

Table 2. Elevated Tumor Markers Highly Suggestive of Testicular Cancer

\begin{tabular}{ll}
\hline Alpha fetoprotein & 2.2 \\
Beta HCG & 902,758 \\
LDH & 5,246 \\
\hline
\end{tabular}

the bleeding of multiple metastatic hemorrhagic lesions. IRguided embolization of hepatic arteries was attempted but despite that hematocrit continued to plummet requiring multiple blood transfusions.

An IR-guided liver biopsy was obtained which showed pure choriocarcinoma (Fig. 4) and due to extensive hemorrhagic lesions a decision was made by oncology to start on chemotherapy with etoposide and cisplatin to contain the bleeding and treat the tumor.

Patient went on to have radicular testicular orchiectomy.

As chemotherapy started, his hemoglobin slowly started returning to normal levels. It was a very rare occasion where hemoglobin could be used as a marker for tumor improvement. Along with it the tumor markers including beta HCG and LDH all showed a decline (Table 3).

Patient also underwent elective radical orchiectomy. The histology from the specimen showed mixed GCT with 50\% choriocarcinoma and 50\% teratoma. Note that the metastatic lesion histology was consistent with pure choriocarcinoma. Metastasis from a mixed GCT as pure choriocarcinoma is also uncommon.

Repeat imaging showed interval reduction in the pulmonary and hepatic metastasis with many lesions showing necro-

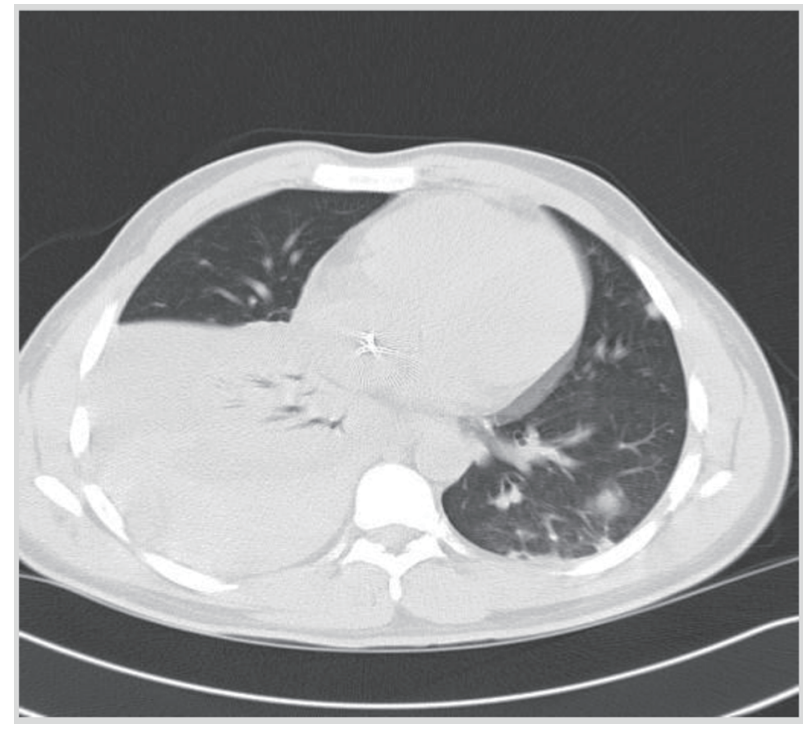

Figure 3. CT chest with large right pleural effusion and metastatic lung nodules. 


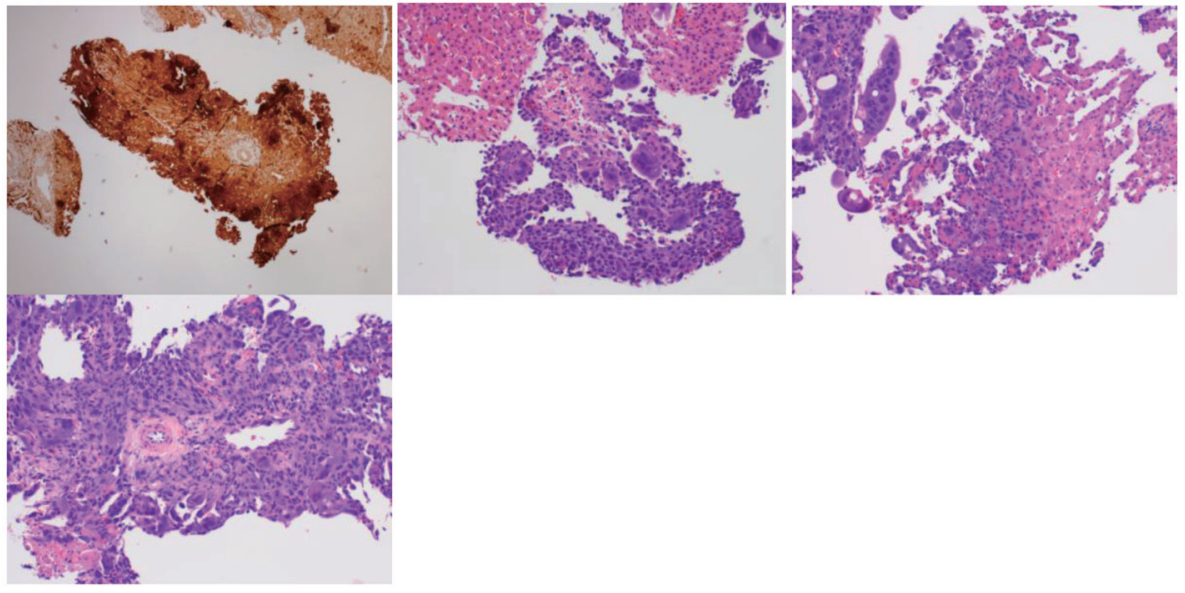

Figure 4. Pathological slides of choriocarcinoma (hematoxylin-eosin and immunohistochemical staining).

sis.

Patient completed first cycle of chemotherapy with good response followed by four more cycles of chemotherapy with cisplatin, bleomycin and etoposide. His beta HCG dropped to 4 , suggestive of near complete remission.

He was referred to a tertiary center and was recommended retroperitoneal lymph node dissection to remove residual disease due to component of teratoma. Histology of surgical specimen showed only necrotic tissue with no evidence of residual tumor. He remains in remission and 3 years since the initial presentation.

\section{Discussion}

Choriocarcinomas are one of the most aggressive and rapidly growing GCTs. In stark contrast to other testicular seminomas and GCTs, it metastasizes rapidly to liver, lungs, brain and other visceral organs. By the time the tumor is symptomatic, the patient has already developed acute complications from hemorrhage or necrosis of the primary tumors or their metastasis or symptoms from high levels of beta HCG.

The tumor occurs most commonly in men aged $20-30$ years with a reported incidence of 2.1 cases per 10,000 males and four times greater incidence in white males than in black males [1-3]. Although it accounts for less than 1\% of all testicular cancers, it is a common component of other testicular GCTs.

Metastasis from mixed GCTs as pure choriocarcinoma is also uncommon like in this case.

The tumor itself may be small and asymptomatic and the first presentation most often is from symptoms of metastatic disease. These include hemoptysis, hematemesis, melena as

Table 3. Decline in Tumor Markers and Stabilization of Hemoglobin as Indicator of Good Response to Chemotherapy

\begin{tabular}{ll}
\hline Beta HCG & 5,260 \\
Hemoglobin & $10 \mathrm{~g}$ \\
\hline
\end{tabular}

well as bleeding into closed spaces like peritoneum, pleura and scrotum [4-7]. The tumor is highly vascular and can bleed severely. In addition, involvement of liver contributes to clotting factor deficiency which aggravates the bleeding [8].

Bleeding could be severe enough at times leading to hemodynamic instability and hemorrhagic shock.

Cryptorchidism is a high risk factor for testicular tumor. Abdominal undescended testis is associated with greater risk than inguinal form. Whereas an abdominal testis cancer is usually a seminoma, a testis fixed to scrotum via orchipexy is more likely to cause non-seminomas GCT (NSGCT) [9].

Genetic changes in the form of amplifications and deletions are observed predominantly in the 12 p11.2-p12.1 chromosomal region. Gain of $12 p$ sequences is associated with invasive growth of seminomas and NSGCTs.

Other risk factors are mumps, trauma and maternal estrogen exposure.

Metastatic NSGCTs are highly sensitive to platinum-based chemotherapy with $80 \%$ cure in advanced stages and $100 \%$ in early stage cancers. Pure choriocarcinoma is extremely rare variant and is not as sensitive as other GCTs but still treated with similar initial chemotherapy as other GCTs. These patients may require salvage chemotherapy regimen and referral to a tertiary cancer center to be treated under various protocols. Cases responsive to chemotherapy may require additional surgical debulking along with radical inguinal orchiectomy.

Logothetis et al (1986) described choriocarcinoma syndrome which includes bleeding complication from metastic sites along with symptoms from grossly elevated beta HCG. This clinical syndrome although is rare but life threatening and requires immediate management like in this case $[10,11]$.

A multimodal approach involving the urologist and hematologist/oncologist is essential in the treatment of advanced NSGCT.

Prior to diagnosis, testicular self-examination on a monthly basis should begin at puberty.

Following diagnosis of testicular carcinoma, a strict adherence to follow-up regimens must be discussed. Initially, the follow-up regimens are more frequent, as tumors can dramatically advance within short intervals. 
Most cases do not carry good prognosis. In a 9-year review of patients treated in multi-agent chemotherapy trials at Memorial Sloan-Kettering Cancer Center, Bosl et al (1983) reported five cases of pure choriocarcinoma and two long-term survivors [12].

This case highlights the importance of recognizing acute manifestations of metastatic choriocarcinoma on initial presentation.

\section{Disclosures}

The authors have no conflict of interest and no funding was obtained.

\section{References}

1. Schottenfeld D, Warshauer ME, Sherlock S, Zauber AG, Leder M, Payne R. The epidemiology of testicular cancer in young adults. Am J Epidemiol. 1980;112(2):232-246.

2. Wingo PA, Tong T, Bolden S. Cancer statistics, 1995. CA Cancer J Clin. 1995;45(1):8-30.

3. Mostofi FK. Proceedings: Testicular tumors. Epidemiologic, etiologic, and pathologic features. Cancer. 1973;32(5):1186-1201

4. Stokes EW, Perkins C. Testicular choriocarcinoma: an unusual presentation as occult gastrointestinal blood loss. J Adoles Health Care. 1989;10:146-150.

5. Benditt JO, Farber HW, Wright J, Karnad AB. Pulmonary hemorrhage with diffuse alveolar infiltrates in men with high-volume choriocarcinoma. Ann Intern Med.
1988;109(8):674-675.

6. Nishizaki T, Orita T, Tsuha M, Wakuta Y, Fujii M, Ito H. Brain metastasis of testicular tumor with massive hemorrhage - report of two cases. Neurol Med Chir (Tokyo). 1991;31:586-589.

7. Kidd D, Plant GT, Scaravilli F, McCartney AC, Stanford M, Graham EM. Metastatic choriocarcinoma presenting as multiple intracerebral haemorrhages: the role of imaging in the elucidation of the pathology. J Neurol Neurosurg Psychiatry. 1998;65(6):939-941.

8. Lox CD, Trevino J. Abnormal hematological indices associated with metastatic choriocarcinoma in a young man. J Med. 1983;14(2):95-101.

9. Batata MA, Whitmore WF, Jr., Chu FC, Hilaris BS, Loh J, Grabstald H, Golbey R. Cryptorchidism and testicular cancer. J Urol. 1980;124(3):382-387.

10. Logothetis CJ, Samuels ML, Selig DE, Ogden S, Dexeus F, Swanson D, Johnson D, et al. Cyclic chemotherapy with cyclophosphamide, doxorubicin, and cisplatin plus vinblastine and bleomycin in advanced germinal tumors. Results with 100 patients. Am J Med. 1986;81(2):219228.

11. Tatokoro M, Kawakami S, Sakura M, Kobayashi T, Kihara K, Akamatsu H. Successful management of lifethreatening choriocarcinoma syndrome with rupture of pulmonary metastatic foci causing hemorrhagic shock. Int J Urol. 2008;15(3):263-264.

12. Bosl GJ, Geller N, Cirrincione C, Hajdu SI, Whitmore W, Jr., Nisselbaum J, Vugrin D, et al. Interrelationships of histopathology and other clinical variables in patients with germ cell tumors of the testis. Cancer. 1983;51(11):21212125 . 